\title{
Presence of cancer cells in the periarterial tissues of patients with advanced gastric cancer
}

\author{
HIROSHI YAMAMOTO ${ }^{1}$, SATOSHI MURATA ${ }^{2,3}$, SACHIKO KAIDA ${ }^{2}$, TSUYOSHI YAMAGUCHI $^{2}$, \\ MITSUAKI ISHIDA $^{4}$, RYOJI KUSHIMA ${ }^{5}$ and MASAJI TANI ${ }^{2}$
}

\author{
${ }^{1}$ Department of Gastrointestinal Surgery, Kusatsu General Hospital, Kusatsu, Shiga 525-8585; ${ }^{2}$ Department of Surgery, \\ Shiga University of Medical Science; ${ }^{3}$ Cancer Center, Shiga University of Medical Science Hospital, Otsu, Shiga 520-2192; \\ ${ }^{4}$ Division of Diagnostic Pathology, Kansai Medical University, Hirakata, Osaka 573-1191; ${ }^{5}$ Department of \\ Clinical Laboratory Medicine, Shiga University of Medical Science, Otsu, Shiga 520-2192, Japan
}

Received October 28, 2017; Accepted April 26, 2018

DOI: $10.3892 / \mathrm{ol} .2018 .8704$

\begin{abstract}
Lymphadenectomy with gastrectomy is considered a curative surgical treatment for gastric cancer. Periarterial connective tissue-preserving lymphadenectomy has become a common procedure following developments in laparoscopic surgery. However, the presence of cancer cells in the periarterial tissue, including neural invasion, has not been examined. In the present study, the periarterial tissues from the vessel roots of the left gastric artery (LGA) and right gastroepiploic artery (RGEA) after gastrectomy were evaluated for the presence of cancer cells. The study included 28 consecutive patients who underwent gastrectomy for gastric cancer. The vessel roots of the RGEA and LGA were obtained from surgically resected specimens and examined by two independent pathologists to determine the presence of cancer cells in the periarterial tissues. The collected specimens included 23 RGEA roots and 26 LGA roots from 28 patients. In 8 cases of early gastric cancer, no cancer cells were indicated in the periarterial tissues. By contrast, cancer cells, including neural invasion in 2 cases, were identified in the periarterial tissues from the roots of examined gastric arteries in 3/20 (15\%) cases of advanced gastric cancer. Notably, all 3 cases featured multiple regional lymph node (LN) metastases. Cancer cells were detected in the perivascular tissue of the major gastric arteries from cases with advanced gastric cancer with LN metastases, suggesting the requirement for oncologic evaluation to ensure adequate vascular tissue margins and an adequate periarterial layer during lymphadenectomy.
\end{abstract}

Correspondence to: Dr Hiroshi Yamamoto, Department of Gastrointestinal Surgery, Kusatsu General Hospital, Yabase 1660, Kusatsu, Shiga 525-8585, Japan

E-mail: yhiroshi@belle.shiga-med.ac.jp

Key words: gastric cancer, cancer cells, lymphadenectomy, neural invasion, periarterial tissue, perivascular connective tissue, surgery, outermost layer

\section{Introduction}

Recent advances in laparoscopic gastrectomy for gastric cancer, including reduced invasiveness and development of standardized procedures, have been beneficial for patients (1). For example, laparoscopic surgical procedures for lymph node (LN) dissection, a well-recognized crucial step in curative gastrectomy for gastric cancer, have been standardized during the past two decades. Classically, complete LN dissection around arterial vessels in cases of advanced gastric cancer, particularly when performed with curative intent, involved exposure of the tunica adventitia (2). However, more recent laparoscopic approaches have enabled surgeons to visualize and recognize the exact borders between the LNs and perivascular tissues, including the neuronal fibers, lymphatic vessels, and fatty tissues that surround arteries. Kanaya et al (3), described this visible layer as the 'outermost layer' and insisted that during lymphadenectomy, it would be beneficial to preserve the perivascular connective tissues surrounding various arteries, including the hepatic and splenic arteries (4). Dissection of suprapancreatic LNs (e.g., 8a and 11) is considered a critical point during lymphadenectomy because damage to the pancreas can lead to major post-gastrectomy complications. Lymphadenectomy procedures that preserve the periarterial connective tissue are therefore considered superior to suprapancreatic $\mathrm{LN}$ dissection in terms of preventing pancreatic damage while removing the LN. Majority of the surgeons in Japan and other countries, therefore adhere to the former procedures, especially for prophylactic LN dissection in cases with early gastric cancer.

During a gastrectomy, the main gastric arteries designated for resection are often bundled and cut along with the periarterial connective tissue, which includes thick nerve fibers. Recently, however, an LN dissection procedure that aims to preserve these periarterial connective tissues has been applied to patients with both early and advanced gastric cancer $(4,5)$. The prevalence and severity of neural invasion from the primary tumor have been shown to correlate significantly with reduced survival among patients with gastrointestinal malignancies such as adenocarcinoma of the esophagogastric junction-II/III and gastric cancer (6). Neural invasion from the primary tumor 
has also been identified as a significant prognostic factor in cases of diffuse invasive gastric cancer (7) as well as gastric cancer after curative resection (8). Currently, the incidence of cancer cell invasion into this periarterial connective tissue, which also comprises of lymphatic vessels and fat tissue, is thought to be almost zero in cases with early gastric cancer. Also, neural invasion into these periarterial tissues is thought to occur infrequently in cases with gastric cancer, compared to other cancers such as hepatobiliary or pancreatic cancer (9). However, no study has investigated the presence of cancer cells in periarterial tissues from patients with gastric cancer.

We conducted the present study to evaluate the safety of lymphadenectomy with periarterial connective tissue preservation for treatment of gastric cancer. Proximal roots of the left gastric artery (LGA) and right gastroepiploic arteries (RGEA) obtained from surgical specimens following gastrectomy were microscopically examined for the presence of cancer cells, including neural invasion in the periarterial tissues.

\section{Materials and methods}

From April 2014 to November 2015, 23 roots of the RGEA and 26 roots of the LGA were collected from 28 consecutive gastric cancer patients and processed for histopathological examination. All patients underwent D1+ or D2 gastrectomy with preservation of the perivascular tissues surrounding the hepatic and splenic artery at the Shiga University of Medical Science Hospital. Case data were collected from our hospital database and clinical records.

We pathologically examined the proximal roots of the LGA and RGEA to detect cancer cells in the periarterial tissues. We sampled the periarterial connective tissues around these arteries which we considered as the distal vascular lymphadenectomy margin. Resected specimens were stained with hematoxylin and eosin according to standard procedures prior to evaluation. Histopathological analysis was performed by 2 independent pathologists (M.I. and R.K.). Histological diagnoses and responses to neoadjuvant chemotherapy were evaluated based on the Japanese guidelines for gastric cancer surgery and pathology (10). The ethics committee in Shiga University of Medical Science approved this retrospective observational study, including the patient's passive consent method and it conformed to the 1995 provisions of the Declaration of Helsinki (as revised in Brazil 2013). Passive consent, commonly termed opt-out consent, assumes agreement to study participation unless consent is deliberately withdrawn. For this type of study, formal consent is not required $(11,12)$.

\section{Results}

The patient characteristics are shown in Table I. The tumor stages of I, II, III and IV were seen in 8, 8, 9 and 3 patients, respectively. We detected cancer cells in the periarterial tissues from $3(15 \%)$ of the 20 advanced gastric cancer cases. These 3 cases were classified as pathological stages IIB, IIIC, and IV, and 2 of them had received neoadjuvant chemotherapy. Cancer cells were detected in the thick fibrous tissue with neural invasion in one case, in fat tissues in the second case, and in fat tissues with neural invasion in the third case. While 2 cases were obtained from specimens surrounding the LGA, one case was from the RGEA. In contrast, cancer cells were not detected in the periarterial tissues from the 8 patients with early gastric cancer.

Neural invasion was observed in the first case of a 61-year-old male patient with esophagogastric junction carcinoma (Siewert type 3 ). This patient underwent total gastrectomy with D2 LN dissection with exposure of the arterial tunica adventitia and received additional hyperthermic intraperitoneal chemotherapy following a diagnosis of T4 advanced gastric cancer with serosal invasion and LN metastasis (SE, N3b, H0, P0, CY0). Following a macroscopic and histopathological examination, the tumor was diagnosed as a carcinoma at the esophagogastric junction that comprised of a well to moderately differentiated tubular adenocarcinoma with serosal invasion (T4a, ly2, v2) and two perigastric LNs (no. $2 ; 1 / 1$, no. 3a; 6/10). Cancer cells and accompanying fibrosis were observed in very close proximity to the arterial wall (Fig. 1). Neural fibers were observed both within and outside the fibrous tissue (Fig. 1), and neural invasion was seen in the periarterial tissue (Fig. 2). The patient received adjuvant chemotherapy with S-1. Metastatic descending colon cancer was detected 27 months after surgery, and he underwent colectomy. Another 10 months later, peritoneal metastasis was detected, and he underwent resection of the small intestine for an obstructive ileus due to peritoneal metastasis. Forty months after surgery, he received best supportive care.

The second case involved a 71-year-old female patient with a T4 advanced gastric cancer located in the middle of the greater curvature of the stomach, with liver invasion (T4b, N0, $\mathrm{H} 0, \mathrm{P} 0)$. She underwent total gastrectomy, splenectomy, and D2 LN dissection with exposure of the arterial tunica adventitia after 2 courses of neoadjuvant S-1/CDDP chemotherapy, as well as partial resection of the lateral segment of the liver. The pathological diagnosis was mucinous adenocarcinoma of the stomach, whereas no carcinoma invasion to the liver was observed. Metastasis was confirmed histologically in 12/26 LNs (no. $1: 2 / 2$, no. 3 a: $1 / 1$, no. 3 b: $2 / 2$, no. 4 d: $1 / 1$, no. $6: 1 / 2$, no. $7: 1 / 1$, no. $9: 2 / 2$, no. $11 \mathrm{~d}: 2 / 2$ ). The final histological diagnosis was gastric carcinoma (UML, type 4, T4a(SE), med, INFc, ly2, v1, P0, H0, M0, N3, CY1, stage IV), and the patient exhibited a grade 1a pathological response to chemotherapy. A specimen from the root of the LGA contained cancer cells in the periarterial fat tissue, with no neural invasion (Fig. 3). Although she received adjuvant chemotherapy with 8 courses of S-1 and oxaliplatin, pleural effusion and ascites were detected which turned out to be peritoneal carcinomatosis. Nine months after surgery, she received a second line of chemotherapy with ramucirumab and paclitaxel. However, eighteen months after surgery she died of peritoneal carcinomatosis.

The third case involved a 79-year-old female patient with gastric cancer located in the lower greater curvature of the stomach. The tumor had invaded the pancreas body and affected the LNs (T4b, N2, H0, P0). After 2 courses of neoadjuvant S-1/CDDP chemotherapy, she underwent total gastrectomy, splenectomy, and D2 LN dissection with exposure of the arterial tunica adventitia, leading to a histological tumor diagnosis of poorly differentiated adenocarcinoma 
Table I. Patient characteristics ( $n=28$ patients).

\section{Variables}

no

Mean age (range)

Sex

Male

Female

Location in stomach

Upper third of stomach

Middle third of stomach

Lower third of stomach

Final gastric cancer stage stage

$$
\text { I }
$$

II

III

IV

pT

T1

$\mathrm{T} 2$

T3

$\mathrm{T} 4$

$\mathrm{pN}$

N0

$\mathrm{N} 1$

$\mathrm{N} 2$

N3

Lymphatic invasion grade$$
0
$$

1

2

3

Vascular invasion grade

$$
0
$$

1

2

3

Neoadjuvant chemotherapy

Yes

No

pT, pathological T; pN, pathological N.

of the stomach. Histologically, 8/20 LN metastases were confirmed (no. $1: 1 / 5$, no. $4 \mathrm{~d}$ : $3 / 6$, no. $6: 3 / 5$, no. $12 \mathrm{a}: 1 / 1$ ). The pathological diagnosis was gastric carcinoma (L, type 3, T2 (MP), sci, INFc, ly1, v0, P0, H0, M0, N2, CY1, stage IV), and the primary tumor exhibited a grade 2 histological response after preoperative chemotherapy. Cancer cells were detected in the periarterial fat tissue, and neural fibers were found in a specimen from the root of the RGEA. She received chemotherapy with 4 courses of S-1 and cisplatin followed by S-1 for 15 months. Twenty-six months after surgery, she is currently in remission.

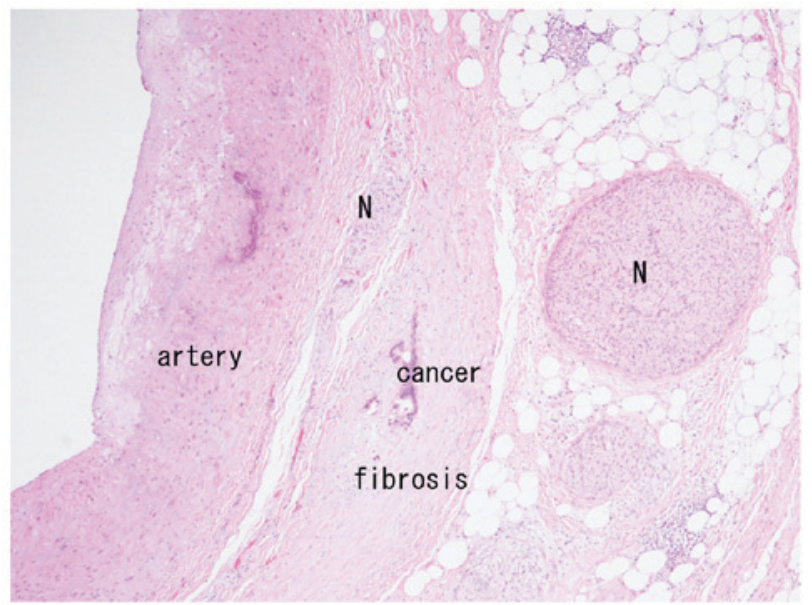

Figure 1. Representative hematoxylin and eosin-stained slide showing the presence of cancer cells in perivascular tissue surrounding the LGA from case 1 . Magnification, $x 40$. N, neural fiber; LGA, left gastric artery.

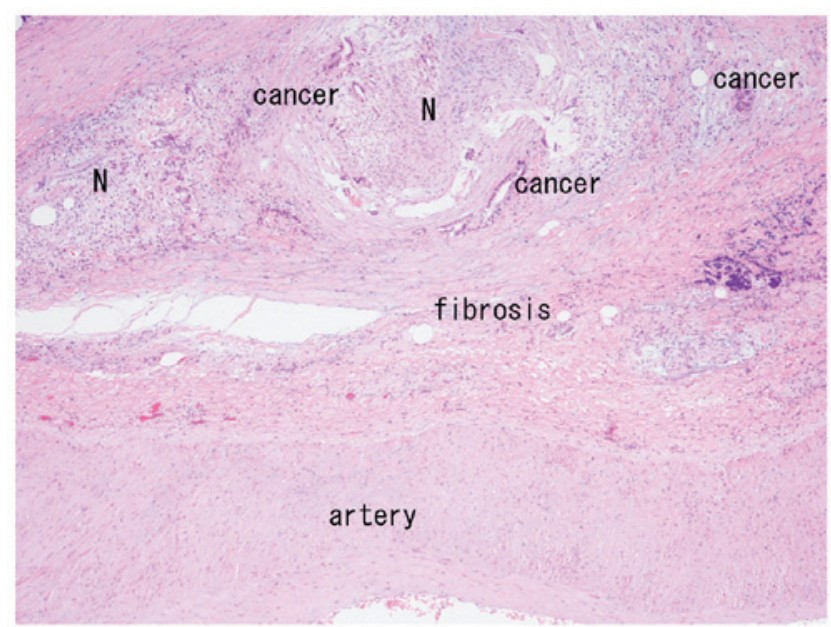

Figure 2. Representative hematoxylin and eosin-stained slide showing the presence of cancer cells including neural invasion in perivascular tissue surrounding the LGA from case 2. Magnification, x40. N, neural fiber; LGA, left gastric artery.

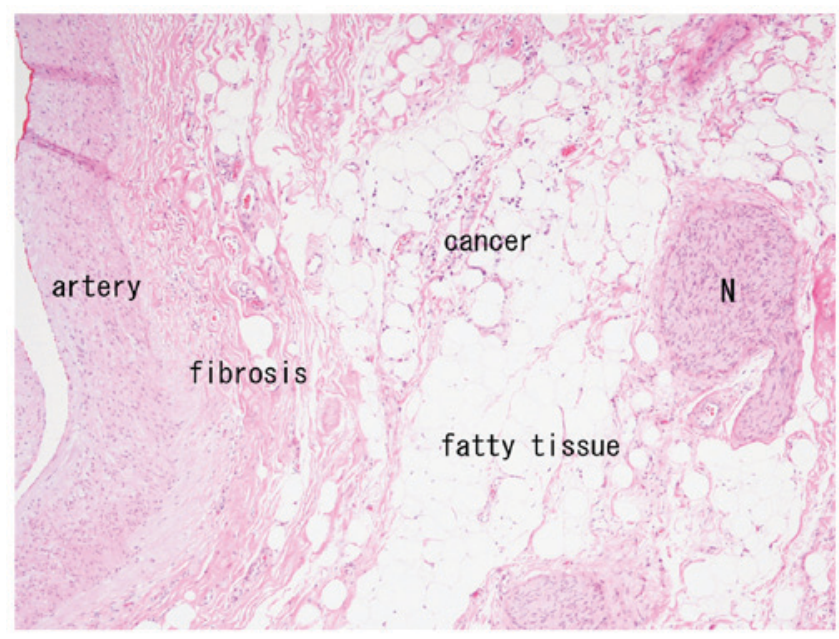

Figure 3. Representative hematoxylin and eosin-stained slide showing the presence of cancer cells in the periarterial fat tissue in perivascular tissue surrounding the LGA from case 3. Magnification, $x 40$. N, neural fiber; LGA, left gastric artery. 


\section{Discussion}

In our study of specimens resected during gastrectomy, we observed cancer cells in the perivascular tissues surrounding the LGA and RGEA of patients with advanced gastric cancer. Although previously perivascular tissue preservation was usually performed in patients undergoing curative gastrectomy with lymphadenectomy for early gastric cancer, extended lymphadenectomy with D2 LN dissection has become popular for patients with advanced gastric cancer (13). However, in Japan, the last 2 decades have seen changes in the actual lymphadenectomy procedure used around the roots of gastric arteries intended for resection and along arterial vessels, including the hepatic and splenic arteries.

In Japan, prior to the popularization of laparoscopic gastrectomy, lymphadenectomy for gastric cancer (particularly advanced gastric cancer) had involved exposure of the arterial tunica adventitia (2). The recent laparoscopic approaches have introduced new surgical anatomy with particular reference to the membrane structures, leading to the establishment of sophisticated lymphadenectomy procedures that preserve the arterial connective tissues of patients with gastric cancer. The designated 'outermost layer' appears to be a reasonable anatomical landmark during lymphadenectomy in patients with early gastric cancer. Indeed, a recent multicenter phase II trial involving patients who underwent laparoscopy-assisted distal gastrectomy with suprapancreatic nodal dissection for clinical stage I gastric cancer found that only $1.7 \%$ of the patients (a low rate) developed an anastomotic leakage or a pancreatic fistula (14). However, we became concerned about the safety of this lymphadenectomy procedure in patients with advanced gastric cancer. Although the main gastric arteries are usually resected at their roots during radical LN dissection for gastric cancers, it was unclear whether the periarterial tissues surrounding the roots of arteries should be totally removed. In the current study, we detected cancer cells in the periarterial tissues surrounding the LG and RGEA roots in $15 \%$ of the patients with advanced gastric cancer and LN metastasis. To the best of our knowledge our study is the first to have employed this approach. This finding suggests that in advanced cases, periarterial tissues should be removed completely under arterial tunica adventitia exposure in order to eliminate any residual cancer cells at the roots of major gastric arteries.

In addition, although we did not present direct evidence, cases of advanced gastric cancer with LN metastasis may also involve periarterial tissues around the hepatic and splenic arteries. A recent paper that described the standardization of D2 lymphadenectomy and surgical quality control used the term 'exposure of vessels' to describe suprapancreatic lymphadenectomy (15). However, previous reports have not distinguished between the preservation and removal of periarterial tissues. We propose that there are two types of curative lymphadenectomy for patients with gastric cancer. One is prophylactic, and the other is therapeutic lymphadenectomy. While the prophylactic lymphadenectomy involves dissection of potentially metastasized LNs, therapeutic lymphadenectomy involves dissection of obviously metastasized LNs. In cases of therapeutic lymphadenectomy, especially those involving advanced gastric cancers, there is no consensus regarding whether periarterial tissues should be preserved or resected. Therefore, future evaluations should address the issues of periarterial cancer invasion and adequate removal of periarterial tissue around the major arteries with the intent to establish radical regional lymphadenectomy along with an oncologically adequate layer, especially for advanced gastric cancers with LN metastases. Importantly, surgeons who perform almost all gastrectomies by the laparoscopic approach should not select the outermost layer for LN dissection of far advanced tumors, especially those with bulky LNs. Several reports on laparoscopic surgery for advanced gastric cancer have emphasized on lymphadenectomy $(4,6,16)$.

Neural invasion by hepatobiliary and pancreatic cancers is a popular research topic with demonstrated prognostic relevance (9). By contrast, neural invasion by gastric cancer was previously thought to be rare but has recently been identified as a critical prognostic factor (6-8). However, neural invasion in the context of gastric cancer was defined within the primary tumor and not in the periarterial tissue, as discussed in the present study. Because the advanced gastric cancers in this study clearly demonstrated the occurrence of neural invasion in the periarterial tissues, future studies will be needed to distinguish the clinical behaviors of neural-invasive cancer cells in periarterial tissues from those within the main tumor.

Pathologically, the effectiveness of gastric surgery has not yet been evaluated based on the residual cancer cells at the lymphadenectomy margins. Our study suggests that periarterial connective tissues around major gastric arteries should be sampled and considered as a distal vascular lymphadenectomy margin in order to pathologically confirm the curability of surgical specimens from advanced gastric cancers. Failure to perform such an evaluation could have potentially led to an underestimation of tumor stage in 3 positive cases in this study. Furthermore, the presence of cancer cells around the vascular margin suggests the potential need for intensive postoperative chemotherapy. However, further studies will be needed to clarify the clinical significance of the residual cancer cells in the vascular margin with periarterial connective tissue invasion. Immunohistochemical staining for claudin 4 has been shown to be useful for detecting a small number of cancer cells (17). However, in the present study, as there were a sufficient number of cancer cells, we were able to detect them via standard hematoxylin and eosin staining without requiring to additionally stain for claudin 4 .

We must note a particular limitation of this study, namely the small number of examined cases. A larger number of patients and additional arterial specimens would have provided more information about cancer cell infiltration around the periarterial connective tissues associated with gastric cancer, particularly in terms of the resection margins of main gastric arteries, the adequate periarterial layer for lymphadenectomy, and pathological distal lymphadenectomy margins.

In conclusion, the present study microscopically demonstrated the presence of cancer cells in periarterial tissues from patients with advanced gastric cancer. Surgeons must, therefore, consider the possibility of cancer cell invasion within preserved periarterial tissues, although the clinical relevance of these cells remains to be determined. Further studies to elucidate the clinical effects of perivascular cancer cells on patient prognosis are warranted. 


\section{Acknowledgements}

Not applicable.

\section{Funding}

No funding was received.

\section{Availability of data and materials}

The datasets used and/or analyzed during the current study are available from the corresponding author on reasonable request.

\section{Authors' contributions}

HY contributed to the study conception and design, HY, SM, SK, TY and MI were involved in the acquisition of data. HY, MI, MT and RK were involved in the analysis and interpretation of data. HY and SM drafted the manuscript and RK and MT critically revised the manuscript.

\section{Ethics approval and consent to participate}

The protocol for this research project was approved by the Ethics Committee of the Shiga University of Medical Science, and it conformed to the 1995 provisions of the Declaration of Helsinki (as revised in Brazil 2013).

\section{Consent for publication}

Not applicable.

\section{Competing interests}

The authors declare that they have no conflict of interest.

\section{References}

1. Koeda K, Nishizuka S and Wakabayashi G: Minimally invasive surgery for gastric cancer: The future standard of care. World J Surg 35: 1469-1477, 2011.

2. Funabiki T: Extended radical subtotal gastrectomy with R3 lymphnodal dissection as well as saccate bursctomy. Jpn J Gastroenterol Surg 24: 162-166, 1991.

3. Kanaya S, Haruta S, Kawamura Y, Yoshimura F, Inaba K, Hiramatsu Y, Ishida Y, Taniguchi K, Isogaki J and Uyama I: Video: Laparoscopy distinctive technique for suprapancreatic lymph node dissection: Medial approach for laparoscopic gastric cancer surgery. Surg Endosc 25: 3928-3929, 2011.
4. Uyama I, Suda K and Satoh S: Laparoscopic surgery for advanced gastric cancer: Current status and future perspectives. J Gastric Cancer 13: 19-25, 2013.

5. Liu B and Lu KY: Neural invasion in pancreatic carcinoma. Hepatobiliary Pancreat Dis Int 1: 469-476, 2002.

6. Shinohara T, Satoh S, Kanaya S, Ishida Y, Taniguchi K, Isogaki J, Inaba K, Yanaga K and Uyama I: Laparoscopic versus open D2 gastrectomy for advanced gastric cancer: A retrospective cohort study. Surg Endosc 27: 286-294, 2013.

7. Liebl F, Demir IE, Mayer K, Schuster T, D'Haese JG, Becker K, Langer R, Bergmann F, Wang K, Rosenberg R, et al: The impact of neural invasion severity in gastrointestinal malignancies: A clinicopathological study. Ann Surg 260: 900-907, 2014.

8. Tanaka A, Yoshikawa H, Okuno K, Koh K, Watatani M, Matsumura E and Yasutomi M: The importance of neural invasion (NI) as a prognostic factor in diffuse invasive gastric cancer. Surg Today 27: 692-695, 1997.

9. Bilici A, Seker M, Ustaalioglu BB, Kefeli U, Yildirim E, Yavuzer D, Aydin FM, Salepci T, Oncel M and Gumus M: Prognostic significance of perineural invasion in patients with gastric cancer who underwent curative resection. Ann Surg Oncol 17: 2037-2044, 2010.

10. Japanese Gastric Cancer Association: Japanese Classification of gastric carcinoma: 3rd English edition. Gastric Cancer 14: 101-112, 2011.

11. Littenberg B and MacLean CD: Passive consent for clinical research in the age of HIPAA. J Gen Intern Med 21: 207-211, 2006.

12. Vellinga A, Cormican M, Hanahoe B, Bennett $\mathrm{K}$ and Murphy AW: Opt-out as an acceptable method of obtaining consent in medical research: A short report. BMC Med Res Methodol 11: 40, 2011.

13. Sakuramoto $\mathrm{S}$, Sasako $\mathrm{M}$, Yamaguchi $\mathrm{T}$, Kinoshita $\mathrm{T}$, Fujii M, Nashimoto A, Furukawa H, Nakajima T, Ohashi Y, Imamura $\mathrm{H}$, et al: Adjuvant chemotherapy for gastric cancer with S-1, an oral fluoropyrimidine. N Engl J Med 357: 1810-1820, 2007.

14. Katai H, Sasako M, Fukuda H, Nakamura K, Hiki N, Saka M, Yamaue H, Yoshikawa T and Kojima K; JCOG Gastric Cancer Surgical Study Group: Safety and feasibility of laparoscopy-assisted distal gastrectomy with suprapancreatic nodal dissection for clinical stage I gastric cancer: A multicenter phase II trial (JCOG 0703). Gastric Cancer 13: 238-244, 2010.

15. Kim HI, Hur H, Kim YN, Lee HJ, Kim MC, Han SU and Hyung WJ: Standardization of D2 lymphadenectomy and surgical quality control (KLASS-02-QC): A prospective, observational, multicenter study [NCT01283893]. BMC Cancer 14: 209, 2014.

16. Fukunaga $\mathrm{T}$, Hiki N, Kubota $\mathrm{T}$, Nunobe $\mathrm{S}$, Tokunaga $\mathrm{M}$, Nohara K, Sano T and Yamaguchi T: Oncologic outcomes of laparoscopy-assisted distal gastrectomy for gastric cancer. Ann Surg Oncol 20: 2676-2682, 2013.

17. Facchetti F, Lonardi S, Gentili F, Bercich L, Falchetti M, Tardanico R, Baronchelli C, Lucini L, Santin A and Murer B: Claudin 4 identifies a wide spectrum of epithelial neoplasms and represents a very useful marker for carcinoma versus mesothelioma diagnosis in pleural and peritoneal biopsies and effusions. Virchows Arch 451: 669-680, 2007. 